\title{
Platelet Membrane $\beta$-Secretase Activity in Mild Cognitive Impairment and Conversion to Dementia: a Longitudinal Study
}

\author{
Bernadette McGuinness*, Marc Fuchs, Suzanne L. Barrett, A. Peter Passmore and Janet A. Johnston \\ Centre for Public Health, School of Medicine, Dentistry and Biomedical Sciences, Queen's University Belfast, \\ N. Ireland
}

\begin{abstract}
A blood-based biomarker to complement the clinical and neuropsychological assessments used to evaluate the risk of individuals with mild cognitive impairment (MCI) developing Alzheimer's disease (AD) would be invaluable. Previous pilot studies by our group identified elevated platelet membrane $\beta$-secretase activity in patients with AD and MCI, as compared to controls, and this activity was influenced by membrane cholesterol levels. The present study investigated baseline platelet membrane $\beta$-secretase activity and cholesterol levels in $97 \mathrm{MCI}$ participants and 85 controls and explored whether these parameters differed in individuals with stable MCI, as compared to those who subsequently developed AD. To evaluate signal specificity, $\beta$-secretase activity assays were conducted in the presence and absence of beta-site amyloid- $\beta$ protein precursor-cleaving enzyme (BACE) inhibitors. Baseline platelet membrane $\beta$-secretase activity did not differ significantly in MCI participants, as compared to controls, and platelet membrane cholesterol levels were significantly lower in the MCI group. The longitudinal study indicated that the activities inhibited by two different BACE inhibitors did not predict conversion to AD; however, the activity that was not affected by BACE inhibitors was significantly (40\%) higher in individuals with stable MCI, as compared with those who subsequently developed AD. These findings indicated that further research into the source of this activity could contribute to a measure facilitating prediction of the risk of conversion from MCI to AD.
\end{abstract}

Keywords: Amyloid- $\beta$ protein precursor, beta-site amyloid- $\beta$ protein precursor-cleaving enzyme, cognition, dementia, Neuropsychology, protease inhibitor

\section{INTRODUCTION}

Mild cognitive impairment (MCI) can be defined as greater cognitive decline than that expected for an individual's age and education level, in the absence of dementia or impairment of activities of daily living (ADLs) [1-2]. In 2004, an international working group on MCI formulated specific recommendations

\footnotetext{
${ }^{*}$ Correspondence to: Dr. Bernadette McGuinness, Room 03.019 Centre for Public Health, Queen's University Belfast, Institute of Clinical Science B, Grosvenor Road, Belfast BT12 6BA, N. Ireland. Tel.: +44 2890 632638; Fax: +44 2890 235900; E-mail: b.mcguinness@qub.ac.uk.
}

for the diagnostic criteria [3] that were employed in the present study. Neuropsychological testing informs the diagnosis of MCI and can assist in delineating specific MCI subtypes. This is important because individuals with amnestic multidomain (AMD) MCI have repeatedly been shown to have a poorer prognosis due to their increased risk for dementia [4-6].

A biomarker to complement the clinical and neuropsychological assessment of individuals with MCI and to assess their risk of conversion to dementia would be extremely useful [7]. The neuropathological changes present in amnestic MCI are similar to those found in $\mathrm{AD}$, with extracellular cortical plaques 
composed of amyloid- $\beta$ peptides $(A \beta)$ and intracellular tangles of abnormally phosphorylated tau [8-10]. The most promising biochemical biomarker combination for the prediction of Alzheimer's disease (AD) in amnestic MCI patients is the measurement of cerebrospinal fluid (CSF) markers of AD pathology, namely $A \beta_{42}$ peptide, total-tau, and phospho-tau levels [11]. However, the use of CSF biomarkers is limited by the invasive nature of lumbar puncture and a bloodbased biomarker would therefore be more useful in a routine clinical setting.

The beta-site amyloid- $\beta$ protein precursor (A $\beta P P)$ cleaving enzymes 1 and 2 (BACE1 and BACE2, referred to collectively as BACE in this paper) catalyze the rate-limiting step in $A \beta$ peptide production, cleaving $A \beta P P$ at the $\beta$-secretase site to generate the $\mathrm{N}$-terminus of $\mathrm{A} \beta$. Our group and others have reported altered levels of BACE and $\beta$-secretase activity in platelets from individuals with AD [12-14]. We identified a significant $17 \%$ increase in platelet membrane $\beta$-secretase activity in $\mathrm{AD}$, which did not correlate with Mini-Mental State Examination (MMSE) score, indicating that this increase did not occur as a secondary result of the disease process and may have preceded symptom onset [14]. Our small pilot study also found $24 \%$ more platelet membrane $\beta$-secretase activity in patients with MCI, as compared to controls [15], while an independent study of a smaller Swedish cohort using different assays found no significant differences in platelet $\alpha$ - or $\beta$-secretase activity in control, MCI, and $\mathrm{AD}$ groups [16]. Membrane $\beta$-secretase activity was found to be influenced by membrane cholesterol levels [17-18]. It should be noted that our previous studies in this area were carried out using a Calbiochem substrate (MCA-EVKMDAEFK-(DNP)-NH2) based on the A $\beta P P$ wild-type sequence at the $\beta$-secretase cleavage site. We therefore refer to this activity as ' $\beta$ secretase', rather than 'BACE' activity, because this type of assay will detect any protease capable of cleaving this peptide sequence and is not specific for the detection of BACE activity. The present study utilized BACE inhibitors to explore whether the observed $\beta$ secretase activity arose from BACE, or from other enzymes capable of cleaving the peptide substrate.

This study examined people who were assessed and diagnosed with MCI shortly after their entry to a memory clinic service. Thorough neuropsychological evaluation was conducted and blood samples were taken to enable a cross-sectional baseline study of membrane $\beta$-secretase activity and membrane cholesterol levels in MCI participants and controls. Within the MCI group, we also analyzed baseline platelet mem- brane parameters in cognitive subgroups. In addition, the MCI group was examined longitudinally and we compared the baseline platelet membrane parameters in individuals who converted to a diagnosis of $\mathrm{AD}$ within 2 years, with those of individuals with stable MCI.

\section{MATERIALS AND METHODS}

\section{Study subjects}

Written informed consent was obtained from all participants, and the study was performed in accordance with local ethical committee procedures (Reference 06/NIR02/55). Individuals with MCI were recruited from the Belfast City Hospital memory clinic. This is a dual consultant-led memory service providing regional diagnosis and treatment; most referrals are received from general practitioners or other specialists. Individuals with MCI usually presented with memory problems but were functionally independent and scored $\geq 24 / 30$ on the MMSE [19] and 82-88/100 on the Addenbrooke's Cognitive Examination-Revised (ACE-R) [20]. Patients were diagnosed with MCI according to criteria developed by an international working group on MCI [3]. Patients of both genders and any age were recruited sequentially as they presented to the clinic. Those with major depressive and other severe psychiatric disorders were excluded, whereas subjects with minor depressive symptoms and mild anxiety were not. MCI participants were not treated with cholinesterase inhibitors and they were not participating in any clinical trial. Controls were recruited from groups of volunteers or were spouses of patients. They had no subjective or objective cognitive complaints and were physically and mentally healthy. All controls were thoroughly interviewed about their physical and mental health prior to inclusion in the study and they were evaluated using the MMSE [19] and ACE-R [20]. Subjects taking any psychoactive medication with a possible impact on cognition (including chronic alcohol or drug abuse) were excluded.

\section{Neuropsychological evaluation}

MCI participants were assessed at a time separate from their clinic visit, usually 2-4 weeks later. The assessment took 45-60 min. As recommended by the American Academy of Neurology, the neuropsychological evaluations assessed speed and attention, learning and episodic memory, visuospatial function, 
language, and executive function. Within each cognitive domain, several aspects of function were assessed using specific tests with demonstrated validity for use within a population with MCI. Briefly, each subject was tested using the National Adult Reading Test (NART) with 50 stimulus words in order to estimate premorbid intellectual status [21]. Episodic memory was tested using paired associate learning (PAL) from the Cambridge neuropsychological test automated battery (CANTAB: http://www.camcog.com/cantabtests.asp) and The New York University immediate and delayed paragraph recall [22]. An executive clock drawing task (CLOX 1) [23] was used to assess executive control, and the Stroop color word test (SCWT) [24] was used to examine attention and response inhibition. The Hayling sentence completion test [25] was used to identify executive effects on initiation speed and response suppression. Color trails A and B [26] were used to assess speed and attention. Language was assessed using the two-part controlled oral word association test (COWAT) [27]. Visuospatial function was assessed using CLOX 2 [23] and the Brixton spatial anticipation test, designed by Burgess and Shallice [25]. Functional ability was measured by the disability assessment in dementia (DAD) [28].

In order to compare control and MCI subjects, standardized z scores (a statistical measurement of a score's relationship to the group mean) adjusted for age and education were calculated for each participant. To differentiate participants with MCI on the basis of the cognitive domains affected, we set a cut-off for each test at 1.5 standard deviation (SD) below the control mean. Designation of this level of impairment as clinically significant was consistent with previous studies of this type [29-30]. Patients with an impaired result on at least one test within a cognitive domain were considered impaired in that domain. MCI participants were then assigned to one of five groups: no impairment of clinical significance (NICS), for individuals who were $<1.5 \mathrm{SD}$ below the control mean for all tests; amnestic single domain (ASD); AMD; non-amnestic single domain (NASD); and non-AMD (NAMD).

\section{Follow-up}

Control subjects were assessed once at baseline and participants with MCI were followed-up annually. The major objective of the follow-up examination was to determine the diagnostic status of the participants (stable MCI, AD, or improved to normal). Diagnosis of AD was based on NINCDS-ADRDA criteria [31]. Those who developed AD were commenced on a cholinesterase inhibitor, if appropriate, and left the study.

\section{Preparation of platelet membrane fractions}

Whole venous blood $(20 \mathrm{~mL})$ was collected in tubes containing ethylenediaminetetraacetic acid and centrifuged ( $150 \mathrm{~g}$, no brake) for $15 \mathrm{~min}$ at room temperature. The platelet-rich plasma supernatant was removed and four volumes of phosphate-buffered saline (PBS; $137 \mathrm{mM} \mathrm{NaCl}, 2.7 \mathrm{mM} \mathrm{KCl}$, and $10 \mathrm{mM}$ phosphate buffer, $\mathrm{pH}$ 7.4) were added. Platelets were recovered by centrifugation $(800 \mathrm{~g}, 5 \mathrm{~min})$; the pellet was suspended in $4 \mathrm{~mL}$ PBS and centrifuged $(800 \mathrm{~g}$, $5 \mathrm{~min}$ ) to obtain a platelet pellet. Platelet pellets were suspended in $1 \mathrm{~mL}$ cell lysis buffer (25 mM HEPES [Sigma], $\mathrm{pH}$ 7.2) and incubated on ice for $1 \mathrm{~h}$ before homogenization by 25-30 strokes of a hand-held safe seal 5-mL homogenizer (Glass Precision Engineering Ltd., Leighton Buzzard, UK) followed by centrifugation $(100,000 \mathrm{~g})$ for $1 \mathrm{~h}$ at $4^{\circ} \mathrm{C}$. The supernatant (cytosolic fraction) was removed and the remaining pellet was suspended in $200 \mu \mathrm{L}$ membrane solubilization buffer $(25 \mathrm{mM}$ HEPES containing $2 \%$ [w/v] CHAPS, Sigma) prior to centrifugation $(20,000 \mathrm{~g})$ for $10 \mathrm{~min}$ at $4^{\circ} \mathrm{C}$. The resulting supernatant (total membrane fraction) was stored at $-80^{\circ} \mathrm{C}$. Sample protein determinations were carried out using a BCA Protein Assay Kit (Pierce, Rockford, IL, USA).

\section{$\beta$-secretase activity assay}

$\beta$-secretase activity was measured using a commercially-available fluorogenic substrate, MCASEVNLDAEFR(DNP)KRR-NH (Sigma A1472 $_{2}$ or Bachem M2465), corresponding to the A $\beta P P$ sequence at the Swedish mutant $\beta$-secretase site, derivatized at its $\mathrm{N}$-terminus with a fluorescent 7-methoxy-4-yl acetyl group (MCA), and at its C-terminus with a 2, 4-dinitrophenyl group (DNP). MCA fluorescence was internally quenched by DNP in the intact peptide, and was detected when the substrate was cleaved. Assays were performed in 96-well black plates and fluorescence was detected using a BMG Labtech FLUOstar OPTIMA (excitation $330 \mathrm{~nm}$, emission $390 \mathrm{~nm}$ ). For the pilot studies, recombinant human BACE1 was obtained from R\&D Systems (931-AS) and native cathepsin D was obtained from Sigma (C8696). Two inhibitors were obtained from Calbiochem; product number 171601 ( $\beta$-secretase inhibitor; KTEETSEVN(stat)VAEF) is referred to as Cal 1 in this study, and product 
number 565788 ( $\beta$-secretase inhibitor IV; a cellpermeable isophthalamide compound containing an hydroxyethylamine motif that binds to BACE1) is referred to as Cal 4.Pilot research investigating the inhibitory profiles of Cal 1 and Cal 4 under our assay conditions showed that they both inhibited the activity of recombinant BACE1, with half-maximal inhibition at concentrations of 100-200 $\mathrm{nM}$ (data not shown). However, these inhibitors also affected the activity of cathepsin D, another aspartyl protease potentially present in the platelet membrane preparations. Cal 1 (100 nM) produced $>90 \%$ inhibition of native cathepsin $\mathrm{D}$, while approximately $5 \mu \mathrm{M}$ Cal 4 was required to achieve half-maximal inhibition of this activity.

For platelet membrane assays, $15 \mu \mathrm{g}$ platelet membrane protein and $5 \mu \mathrm{M}$ MCA-SEVNLDAEFR (DNP)KRR-NH $\mathrm{N}_{2}$ were incubated in a final volume of $100 \mu \mathrm{L}$ in assay buffer $(50 \mathrm{mM}$ sodium actetate, $\mathrm{pH}$ 4.5). The amount of membrane solubilization buffer present in each assay well was standardized at $10 \mu \mathrm{L}$ to ensure that any detergent effects did not influence the assay results. Each platelet sample was assayed in duplicate or triplicate under three assay conditions: 1 ) in the absence of inhibitor, 2) in the presence of $2 \mu \mathrm{M}$ Cal 1 , and 3 ) in the presence of $1 \mu \mathrm{M} \mathrm{Cal} 4$. Our pilot data indicated that at these concentrations, Cal 1 would be expected to inhibit BACE and cathepsin D activities by $>90 \%$, whereas $\mathrm{Cal} 4$ would be expected to inhibit BACE activity by $>90 \%$, without affecting cathepsin D activity. Substrate hydrolysis was followed by measuring fluorescence every $120 \mathrm{~s}$ at $37^{\circ} \mathrm{C}$. Initial rates were calculated over the linear phase (typically for $30 \mathrm{~min}$ ) and converted to [MCA] liberated using a calibration curve on the plate, constructed from a dilution series of 7-methoxycoumarin-4-acetic acid (Sigma). The initial rates were then expressed as pmoles MCA detected per $\mathrm{h}$ per $\mu \mathrm{g}$ membrane protein ( $\mathrm{pmol} \mathrm{MCA} / \mathrm{h} / \mu \mathrm{g}$ protein).

\section{Membrane cholesterol assay}

Platelet membrane fraction cholesterol levels were determined using the Amplex Red Cholesterol Assay Kit (Invitrogen), according to the manufacturer's instructions. Fifty microliters of cholesterol standards (0-1000 pmol per well) or sample triplicates were added to 96-well black plates (Greiner). Fifty microliters of working solution $(300 \mu \mathrm{M}$ Amplex Red reagent, 2 unit $/ \mathrm{mL}$ horseradish peroxide, 2 unit $/ \mathrm{mL}$ cholesterol oxidase, 0.2 unit $/ \mathrm{mL}$ cholesterol esterase) were added. The plate was incubated for $30 \mathrm{~min}$ at $37^{\circ} \mathrm{C}$ in the dark before measurement of fluorescence (excitation $560 \mathrm{~nm}$, emission $590 \mathrm{~nm}$; BMG Labtech FLUOstar OPTIMA). Sample fluorescence was converted to picomoles cholesterol using the standard curve, and results expressed as picomol cholesterol $/ \mu \mathrm{g}$ protein.

\section{Statistical analysis}

Data were analyzed using the Statistical Package for the Social Sciences Version 17 (SPSS 17) for Windows (SPSS Inc., Chicago, IL, USA). Data are presented as mean \pm SD. Study group data were compared using independent Student's $t$-tests, analysis of variance (ANOVA; continuous dependent variables), and $\chi^{2}$ tests (frequency data). The primary endpoint was platelet membrane $\beta$-secretase activity and a power calculation based on our previous pilot study of MCI and control subjects [15] indicated that the numbers recruited in the present study would provide $>90 \%$ power to detect a difference in means of 0.07 using two-tailed tests with a $p$-value of $<0.05$. The baseline platelet parameters were analyzed using a receiver operating characteristic (ROC) curve, to explore their usefulness in discriminating between the study groups.

\section{RESULTS}

\section{Study subjects}

Study subject information is presented in Table 1 . There were no significant differences between the MCI and control groups in terms of age, gender, years of education, premorbid IQ (as determined by

Table 1

Baseline characteristics of study participants

\begin{tabular}{lccc}
\hline & Control $n=85$ & MCI $n=97$ & $p$ \\
\hline Age (years) & $74.6 \pm 9.5$ & $72.4 \pm 9.3$ & 0.11 \\
Age range & $52-94$ & $43-93$ & \\
Gender (\%F:M) & $54: 46$ & $64: 36$ & 0.17 \\
Years of education & $11.6 \pm 3.2$ & $12.2 \pm 2.9$ & 0.15 \\
NART IQ & $113.0 \pm 8.5$ & $113.9 \pm 8.3$ & 0.49 \\
DAD & $79.6 \pm 2.3$ & $79.6 \pm 0.9$ & 0.93 \\
MMSE & $29.3 \pm 0.8$ & $27.9 \pm 1.7$ & $<\mathbf{0 . 0 1}$ \\
ACE-R & $92.1 \pm 2.9$ & $83.2 \pm 8.7$ & $\mathbf{< 0 . 0 1}$ \\
\hline
\end{tabular}

Data provided are mean \pm SD. There were no significant differences in age, gender, years of education, premorbid IQ (National Adult Reading Test; NART) or activities of daily living (disability assessment in dementia; DAD) in the two study groups. The mild cognitive impairment (MCI) group had significantly lower scores in the MiniMental State Examination (MMSE) and Addenbrooke's Cognitive Examination-Revised (ACE-R), as compared with controls. $p$-values are indicated for the comparison between the MCI and control groups by unpaired $t$-test (significant $p$-values are shown in bold). 
NART), or daily function (measured by DAD). The MCI group had significantly lower MMSE and ACE-R scores than the control group. Two of the MCI participants had insufficient neuropsychological data to allow assignment to a neurocognitive domain subgroup. With respect to the remaining $95 \mathrm{MCI}$ participants, $46 \%$ were assigned to the AMD subgroup $(n=44), 18 \%$ to the NASD group $(n=17), 22 \%$ to the NICS group $(n=21), 7 \%$ to the ASD group $(n=7)$, and $6 \%$ to the NAMD group $(n=6)$. MCI patients were followed for $531.3 \pm 308.6($ mean \pm SD) days, with a median follow-up of 510 days (interquartile range 303-774 days). The minimum follow-up period for the subjects included in the present study was 1 year (for subjects who had converted to $\mathrm{AD}$ at 1 year and therefore left the study).

Figure 1 details participant flow through the study. Seven of the 97 MCI participants improved to normal during the two-year follow-up period. Clinical follow-up data was unavailable for 9 MCI participants and these individuals were therefore excluded from further analyses; a participant diagnosed with $\mathrm{VaD}$ was also excluded. Thirty individuals had converted to a diagnosis of $\mathrm{AD}$ by the end of year 2 and 50 individuals had stable MCI. The annual conversion rate to dementia was $15.7 \%$ per year. There was no significant difference in gender between stable MCI and AD converters. Those who converted to $\mathrm{AD}$ were significantly older and had a lower MMSE at baseline than those who remained stable (Table 2).

\section{Platelet membrane $\beta$-secretase activity at baseline}

Platelet membrane $\beta$-secretase activity data are presented in Table 3 as the mean initial rate in the absence of inhibitor (total activity); mean initial rates in the presence of $2 \mu \mathrm{M}$ Cal 1 or $1 \mu \mathrm{M} \mathrm{Cal} \mathrm{4}$; and the activity that was inhibited by each inhibitor (total activity minus the initial rate in the presence of inhibitor). Although all platelet membrane $\beta$-secretase activities tended to be slightly higher in the MCI group (103-114\% of control values), there were no statistically significant differences between the groups. As noted previously, there were large inter-individual differences in activity within the study groups [14]. There was no statistically significant relationship between platelet membrane $\beta$-secretase activity and age or MMSE.

We also subtracted the initial rate observed in the presence of Cal 1 from that observed in the presence

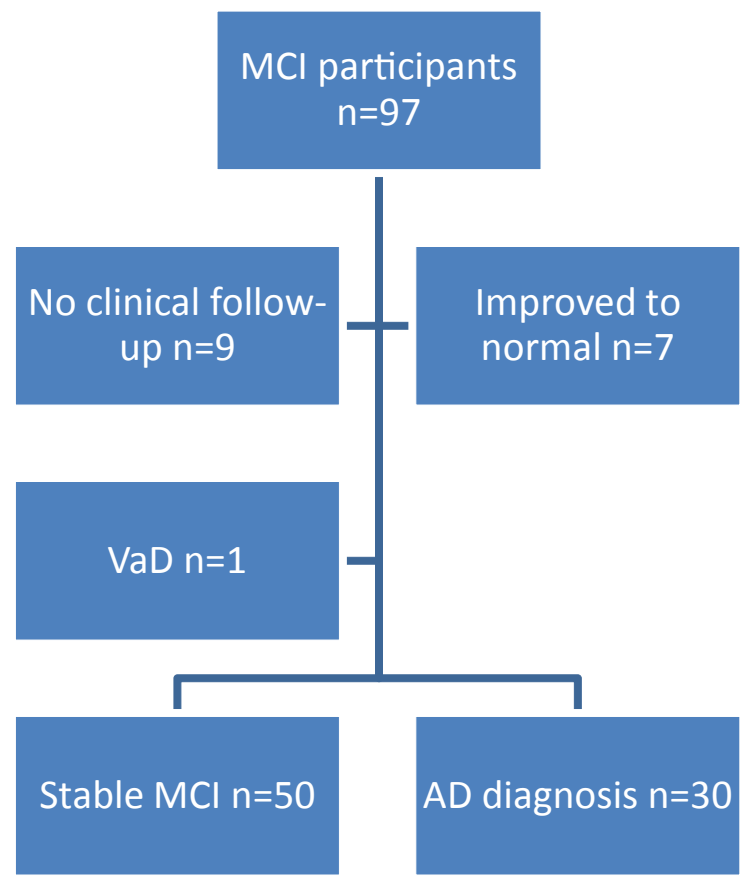

Fig. 1. Participant flow through the study. MCI, mild cognitive impairment; VaD, vascular dementia; AD, Alzheimer's disease

Table 2

Baseline characteristics of study participants who remained stable or converted to Alzheimer's disease (AD)

\begin{tabular}{lccc}
\hline & Stable MCI & Converted to AD & $p$ \\
& $\mathrm{n}=50$ & $\mathrm{n}=30$ & \\
\hline Age & $70.1 \pm 9.9$ & $77.2 \pm 5.6$ & $<\mathbf{0 . 0 1}$ \\
MMSE & $28.3 \pm 1.65$ & $27.0 \pm 1.4$ & $<\mathbf{0 . 0 1}$ \\
Gender (\%F:M) & $60: 40$ & $77: 23$ & 0.10 \\
\hline
\end{tabular}

Data provided are mean \pm SD. The study subjects who converted to AD were significantly older and had significantly lower MiniMental State Examination (MMSE) scores at baseline. $p$-values are indicated for the comparison between the MCI and control groups by unpaired $t$-test (significant $p$-values are shown in bold).

of $\mathrm{Cal} 4$ to provide a measure of the activity of enzymes other than BACE that were inhibited by Cal 1 (for example, cathepsin D). This activity showed no significant difference between the study groups $(0.15 \pm 0.1$ $\mathrm{pmol} \mathrm{MCA} / \mathrm{h} / \mu \mathrm{g}$ protein in both the MCI and control group).

Platelet membrane $\beta$-secretase activity was compared across the MCI cognitive sub-groups. No significant differences in activity were seen between the MCI subgroups represented in this study, as illustrated in Table 4. 
Table 3

Platelet membrane $\beta$-secretase activity

\begin{tabular}{lccc}
\hline \multicolumn{4}{c}{ Initial rate $(\mathrm{pmol} \mathrm{MCA} / \mathrm{h} / \mu \mathrm{g})$} \\
\hline Inhibition & $\begin{array}{c}\text { Control } \\
(n=85)\end{array}$ & $\begin{array}{c}\text { MCI } \\
(n=97)\end{array}$ & $p$ \\
\hline No inhibitor & $0.74 \pm 0.39$ & $0.81 \pm 0.55$ & 0.30 \\
Cal 1 $(2 \mu \mathrm{M})$ & $0.45 \pm 0.30$ & $0.51 \pm 0.33$ & 0.22 \\
Cal 4 $(1 \mu \mathrm{M})$ & $0.60 \pm 0.31$ & $0.65 \pm 0.42$ & 0.32 \\
Inhibited activity & & & \\
No inhibitor - 2 $\mu \mathrm{M} \mathrm{Cal} \mathrm{1}$ & $0.29 \pm 0.17$ & $0.30 \pm 0.32$ & 0.69 \\
No inhibitor - 1 $\mu \mathrm{M}$ Cal 4 & $0.14 \pm 0.10$ & $0.16 \pm 0.19$ & 0.41 \\
\hline
\end{tabular}

Platelet membrane fractions were assayed in duplicate or triplicate, as described in the Methods section: in the absence of inhibitor, in the presence of $2 \mu \mathrm{M} \mathrm{Cal} 1$, or in the presence of $1 \mu \mathrm{M} \mathrm{Cal} \mathrm{4.} \mathrm{Data} \mathrm{are}$ presented as mean initial rate \pm SD under the conditions indicated. The inhibited activity data provides the difference between the initial rates under the indicated conditions. $p$-values are indicated for the comparison between the mild cognitive impairment (MCI) and control groups by unpaired $t$-test with Welch's correction (because the variances differed significantly).

\section{Platelet membrane cholesterol levels and $\beta$-secretase/cholesterol ratios at baseline}

The data presented in Table 5 indicated that platelet membrane cholesterol was $12 \%$ lower in the MCI group (unpaired $t$-test, $p=0.01$ ). For each individual, the ratio of membrane $\beta$-secretase activity to membrane cholesterol level was then calculated (Table 5). In the MCI group, the mean ratio of total membrane $\beta$-secretase activity to membrane cholesterol level was $124 \%$ of that observed in the control group and this difference was statistically significant $(p=0.02$, unpaired $t$-test).

We explored whether this difference could be attributed to BACE activity by calculating the ratio of the portion of enzyme assay activity inhibited by $\mathrm{Cal}$ 1 or $\mathrm{Cal} 4$ to the corresponding membrane cholesterol level. These data are presented in Table 5. Interestingly, the ratios of the Cal 1- or Cal 4-inhibited activities to membrane cholesterol did not differ significantly between the study groups. However, the ratios of the initial rates recorded in the presence of either Cal 1 or Cal 4 (i.e., the activity that was not inhibited by these BACE inhibitors) to membrane cholesterol did show significant differences between the groups. These MCI group values were $131 \%(\mathrm{Cal} 1)$ or $125 \%(\mathrm{Cal} 4)$ that of the control values $(p=0.01$ for both comparisons, unpaired $t$-test).

The ratio of total membrane $\beta$-secretase activity to membrane cholesterol produced a ROC AUC of 0.63 $(p=0.004)$. Similarly, the ROC AUC of the Cal 1uninhibited activity/membrane cholesterol ratio was 0.64 ( $p=0.001)$. ROC analyses of platelet membrane $\beta$-secretase activity measures alone produced a maximum AUC of 0.56 (for the initial rate in the presence of Cal $1 ; p=0.17)$. Membrane cholesterol alone produced a ROC curve AUC of $0.60(p=0.02)$.

\section{Baseline platelet membrane parameters and prognosis}

Table 6 shows the mean platelet membrane parameters for the sub-groups of individuals with MCI who remained stable or converted to AD. Analyses of total $\beta$-secretase activity, membrane cholesterol, and the ratio between them showed no significant differences in the stable MCI group, as compared with the AD group. However, $\beta$-secretase activity in the presence of Cal 1 was significantly different in these groups, as was the ratio of this activity to membrane cholesterol; ROC analyses of these measures produced AUCs of $0.63(p=0.06)$ and $0.64(p=0.04)$, respectively. None

Table 4

Platelet membrane $\beta$-secretase activity in mild cognitive impairment (MCI) subgroups

\begin{tabular}{|c|c|c|c|c|c|c|}
\hline \multicolumn{7}{|c|}{ Initial rate (pmol MCA/h/ $\mu \mathrm{g}$ ) } \\
\hline Inhibition & $\begin{array}{l}\text { NICS } \\
n=21\end{array}$ & $\begin{array}{l}\text { ASD } \\
n=7\end{array}$ & $\begin{array}{l}\text { AMD } \\
n=44\end{array}$ & $\begin{array}{l}\text { NASD } \\
n=17\end{array}$ & $\begin{array}{c}\text { NAMD } \\
n=6\end{array}$ & $\begin{array}{c}\text { ANOVA } \\
p\end{array}$ \\
\hline No inhibitor & $0.97 \pm 0.83$ & $0.85 \pm 0.37$ & $0.78 \pm 0.49$ & $0.79 \pm 0.49$ & $0.62 \pm 0.25$ & 0.13 \\
\hline Cal $1(2 \mu \mathrm{M})$ & $0.63 \pm 0.51$ & $0.59 \pm 0.30$ & $0.45 \pm 0.24$ & $0.54 \pm 0.25$ & $0.48 \pm 0.12$ & 0.28 \\
\hline $\mathrm{Cal} 4(1 \mu \mathrm{M})$ & $0.80 \pm 0.66$ & $0.72 \pm 0.31$ & $0.61 \pm 0.32$ & $0.66 \pm 0.30$ & $0.51 \pm 0.21$ & 0.41 \\
\hline \multirow{3}{*}{$\begin{array}{l}\text { Inhibited activity } \\
\text { No inhibitor }-2 \mu \mathrm{M} \mathrm{Cal} 1 \\
\text { No inhibitor }-1 \mu \mathrm{M} \mathrm{Cal} 4\end{array}$} & & & & & & \\
\hline & $0.34 \pm 0.34$ & $0.25 \pm 0.10$ & $0.33 \pm 0.38$ & $0.25 \pm 0.14$ & $0.21 \pm 0.09$ & 0.78 \\
\hline & $0.17 \pm 0.19$ & $0.13 \pm 0.07$ & $0.17 \pm 0.24$ & $0.13 \pm 0.07$ & $0.11 \pm 0.05$ & 0.53 \\
\hline
\end{tabular}

Individuals were categorized into MCI subgroups as described in the Methods section: NICS, no impairment of clinical significance; ASD, amnestic single domain; AMD, amnestic multiple domain; NASD, non-amnestic single domain; and NAMD, non-amnestic multiple domain. Platelet membrane fractions were assayed in duplicate or triplicate: in the absence of inhibitor, in the presence of $2 \mu \mathrm{M}$ Cal 1 , or in the presence of $1 \mu \mathrm{M} \mathrm{Cal} 4$. Data are presented as mean initial rate \pm SD under the conditions indicated. The inhibited activity data provides the difference between the initial rates under the indicated conditions. $p$-values are indicated for the comparison between the subgroups by analysis of variance (ANOVA). 
Table 5

Platelet membrane cholesterol and $\beta$-secretase activity

\begin{tabular}{|c|c|c|c|}
\hline & Control $n=84$ & MCI $n=89$ & $\mathrm{p}$ \\
\hline Membrane cholesterol ( $\mathrm{pmol} / \mu \mathrm{g}$ protein) & $269.8 \pm 86.3$ & $237.5 \pm 80.0$ & $\overline{0.01}$ \\
\hline Total initial rate/membrane cholesterol & $2.97 \pm 1.8$ & $3.68 \pm 2.1$ & 0.02 \\
\hline Initial rate with $2 \mu \mathrm{M}$ Cal $1 /$ membrane cholesterol & $1.83 \pm 1.3$ & $2.39 \pm 1.5$ & 0.01 \\
\hline (No inhibitor- $2 \mu \mathrm{M}$ Cal 1) initial rate/membrane cholesterol & $1.15 \pm 0.89$ & $1.29 \pm 1.0$ & 0.31 \\
\hline Initial rate with $1 \mu \mathrm{M} \mathrm{Cal} 4 /$ membrane cholesterol & $2.40 \pm 1.4$ & $3.00 \pm 1.7$ & 0.01 \\
\hline (No inhibitor- $1 \mu \mathrm{M} \mathrm{Cal} \mathrm{4)} \mathrm{initial} \mathrm{rate/membrane} \mathrm{cholesterol}$ & $0.58 \pm 0.5$ & $0.68 \pm 0.6$ & 0.23 \\
\hline
\end{tabular}

Platelet membrane fractions were assayed in triplicate for cholesterol and in duplicate or triplicate for enzyme activity, as described in the Methods section: in the absence of inhibitor, in the presence of $2 \mu \mathrm{M} \mathrm{Cal} \mathrm{1,} \mathrm{or} \mathrm{in} \mathrm{the} \mathrm{presence} \mathrm{of} 1 \mu \mathrm{M}$ Cal 4 . Data are presented as the mean \pm SD of the mean initial rate/mean membrane cholesterol level for each individual (multiplied by 1000). $p$-values are indicated for the comparison between the mild cognitive impairment (MCI) and control groups by unpaired $t$-test (significant $p$-values are shown in bold).

Table 6

Predictive value of baseline platelet measures.

\begin{tabular}{|c|c|c|c|c|}
\hline & Stable MCI $n=50$ & Converted to AD AUC (p) $n=30$ & $\mathrm{p}$ & ROC AUC (p) \\
\hline Membrane cholesterol (pmol/ $\mu \mathrm{g}$ protein) & $239.2 \pm 77.6$ & $240.5 \pm 85.5$ & 0.95 & \\
\hline Total initial rate (no inhibitor) & $0.90 \pm 0.61$ & $0.77 \pm 0.52$ & 0.34 & \\
\hline Initial rate with Cal $1(2 \mu \mathrm{M})$ & $0.59 \pm 0.40$ & $0.42 \pm 0.19$ & $\mathbf{0 . 0 1}^{\#}$ & $0.63(0.06)$ \\
\hline Initial rate with $\mathrm{Cal} 4(1 \mu \mathrm{M})$ & $0.74 \pm 0.50$ & $0.58 \pm 0.27$ & $0.06^{\#}$ & $0.59(0.19)$ \\
\hline \multicolumn{5}{|l|}{ Inhibited activity } \\
\hline No inhibitor $-2 \mu \mathrm{M}$ Cal 1 & $0.31 \pm 0.25$ & $0.34 \pm 0.46$ & $0.68^{\#}$ & \\
\hline No inhibitor $-1 \mu \mathrm{M} \mathrm{Cal} 4$ & $0.15 \pm 0.14$ & $0.19 \pm 0.29$ & $0.56^{\#}$ & \\
\hline
\end{tabular}

Data obtained from analyses of baseline blood samples in the mild cognitive impairment (MCI) group were sub-divided depending on whether the individual remained stable or was diagnosed with Alzheimer's disease (AD) over the subsequent 2 years. Seven individuals who became cognitively normal within the same time period were omitted from this analysis. Platelet membrane fractions were assayed in triplicate for cholesterol and in duplicate or triplicate for enzyme activity, as described in the Methods section: in the absence of inhibitor, in the presence of $2 \mu \mathrm{M} \mathrm{Cal} 1$, or in the presence of $1 \mu \mathrm{M} \mathrm{Cal} 4$. Data are presented as the mean \pm SD. $p$-values are indicated for the comparison between the stable $\mathrm{MCI}$ and $\mathrm{AD}$ group, unpaired $t$-test (\# with Welch's correction where variances differed significantly). ROC, receiver operating characteristic; AUC, area under the curve.

of the other measures produced statistically significant results in this respect (Table 6).

\section{DISCUSSION}

This study extended our previous work relating to platelet membrane $\beta$-secretase activity in MCI and $\mathrm{AD}$ by exploring whether similar effects were observed with a different $\beta$-secretase substrate, in the presence and absence of BACE inhibitors, in a longitudinal study of a well-characterized group of individuals with MCI. The current study found that total enzyme activity measured using the $\beta$-secretase activity assay tended to be $10 \%$ higher in platelet membranes from MCI participants, as compared to controls, but this difference was not statistically significant. This contrasted with our earlier pilot study where platelet membrane $\beta$-secretase activity was determined using a different BACE substrate (MCA-EVKMDAEFK-(DNP)- $\mathrm{NH}_{2}$ ) and found to be $24 \%$ higher in MCI participants than in controls $(p=0.001)$ [15]. In addition to the different $\beta$-secretase assay employed, we included greater numbers of MCI participants $(n=97)$ and controls $(n=85)$ in the present study, as compared to the previous one (52 MCI and 75 controls), and they were all characterized much more thoroughly from a neuropsychological perspective. One previous study also reported that platelet $\beta$-secretase activity was not elevated in MCI [16]. However, only 6 MCI participants were assessed in this study, alongside 30 healthy controls and 20 participants with probable AD. Different platelet preparation and $\beta$-secretase assay methods were used and the demographics of the sample populations also differed, making it difficult to compare these studies directly. A more recent study enrolled 34 ASD MCI participants, 45 AD patients, and 28 controls and assayed platelet $\beta$-secretase activity using a Calbiochem substrate with the Swedish mutation sequence. This identified significantly increased activity in the MCI and $\mathrm{AD}$ groups, as compared to age-matched controls (1.9- and 1.5-fold change respectively), with no significant difference between the MCI and AD groups [32].

These previous platelet $\beta$-secretase studies did not employ inhibitors to explore the specificity of the assay signal obtained. The present study investigated the assay signal further by measuring activity in the presence and absence of two BACE inhibitors. This provided a more specific measure of enzyme activity, although this approach was still imperfect due the 
lack of inhibitor specificity. For example, our pilot data indicated that cathepsin $\mathrm{D}$, another aspartyl protease likely to be present in platelet membrane preparations, cleaved the substrate and was also inhibited by the BACE inhibitors to different extents. It is clear that other proteases capable of cleaving this substrate (and the wild-type substrate employed previously) may also be present in the platelet membrane preparations. We therefore referred to the activity reported in this and previous publications as ' $\beta$-secretase', rather than 'BACE', activity. Development of extremely specific non-peptidic BACE1 inhibitors that can block the enzyme activity in complex samples, such as brain lysates, may facilitate more specific measurement of BACE1 activity in the future [33]. Nonetheless, all of the $\beta$-secretase activity measured in assays like the one employed in the present study may be biologically relevant, and the possibility of cathepsin D contributing to platelet $\beta$-secretase activity along with BACE1 has been raised in a review of the relevant literature [33].

Platelet membrane cholesterol levels were significantly lower in the MCI group than in the control group. This was consistent with our previous report that cognitively impaired participants (with AD and MCI) had $18 \%$ lower membrane cholesterol levels than the control group [18]. The present study found that the ratio of total membrane $\beta$-secretase activity/membrane cholesterol was significantly elevated in the MCI group, even though the different study subject groups (MCI versus MCI plus AD) and $\beta$-secretase assays employed meant that it was not possible to compare these studies directly. Interestingly, the use of inhibitors enabled us to determine that there was no significant difference between the ratio of 'specific' BACE activity (no inhibitor - the $\mathrm{Cal} 1$ or Cal 4 initial rates) to membrane cholesterol level in the MCI and control groups. However, the initial rate in the presence of $\mathrm{Cal} 1$ or $\mathrm{Cal} 4 /$ membrane cholesterol level showed the most significant differences between the study groups ( $31 \%$ or $24 \%$ higher in the MCI group, respectively, $p=0.01$ for both); these measures reflected a difference in a $\beta$-secretase-like activity that was not inhibited by the BACE inhibitors.

It is likely that disease-modifying treatments for $\mathrm{AD}$ need to be initiated before overt symptoms are apparent, as these functional changes reflect severe and currently irreversible neurodegenerative changes. A method to identify those at heightened risk for AD in the future is therefore urgently required so that novel therapies can be targeted effectively. Using the longitudinal clinical data, we explored whether the baseline platelet measures could help to identify individuals within the MCI group who would subsequently develop AD. We found that the $\beta$-secretase assay initial rate in the presence of Cal 1 was significantly (40\%) higher in the stable MCI group, and use of the ratio between this enzyme activity and membrane cholesterol produced a ROC AUC of $0.64(p=0.04)$. Although this value indicated that this individual test was a poor discriminator of individuals who would convert from MCI to AD within 2 years, further refinement may enable it to contribute to a prognostic battery used to inform treatment decisions.

Further biochemical analyses with the aim of identifying the source of the enzyme activity measured under these assay conditions would be very valuable as they may help to improve the specificity of this measurement. Although the MCI versus control group comparisons revealed higher levels of enzyme activity and activity/cholesterol ratios in the MCI group as a whole, those MCI subjects who subsequently converted to $\mathrm{AD}$ actually had lower levels of enzyme activity and activity/cholesterol ratios than those who remained stable. Any speculation about the significance of this is difficult, given the lack of data in the area; however, it is possible that these platelet parameters and the relationships between them fluctuate over the course of the disease.

These findings indicated that further research into the source of the difference in platelet membrane $\beta$-secretase activity in individuals with stable MCI and those who converted to $\mathrm{AD}$ has the potential to contribute to the identification of patients who are at increased risk for dementia. The development of prognostic instruments combining neuropsychological information with platelet biochemical measures and known genetic risk factors may facilitate effective targeting of future disease-modifying therapies for AD.

\section{ACKNOWLEDGMENTS}

We are extremely grateful to the American Federation of Aging Research and Atlantic Philanthropies for their award of a Paul Beeson Career Development Award to BMcG. We thank Ryan Connolly for his contribution to the cholesterol assays employed in this study and Professor Chris Patterson for his statistical expertise.

Authors' disclosures available online (http://j-alz. com/manuscript-disclosures/15-0795).

\section{REFERENCES}

[1] Burns, A, Zaudig, M (2002) Mild cognitive impairment in older people. Lancet 360, 1963-1965. 
[2] Gauthier S, Reisberg B, Zaudig M, Petersen RC, Ritchie K, Broich K, Belleville S, Brodaty H, Bennett D, Chertkow H, Cummings JL, de Leon M, Feldman H, Ganguli M, Hampel H, Scheltens P, Tierney MC, Whitehouse P, Winblad B, International Psychogeriatric Association Expert Conference on mild cognitive impairment (2006) Mild cognitive impairment. Lancet 367, 1262-1270.

[3] Winblad B, Palmer K, Kivipelto M, Jelic V, Fratiglioni L, Wahlund LO, Nordberg A, Backman L, Albert M, Almkvist O, Arai H, Basun H, Blennow K, de Leon M, DeCarli C, Erkinjuntti T, Giacobini E, Graff C, Hardy J, Jack C, Jorm A, Ritchie K, van Duijn C, Visser P, Petersen RC (2004) Mild cognitive impairment-beyond controversies, towards a consensus: Report of the international working group on mild cognitive impairment. J Intern Med 256, 240-246.

[4] Summers MJ, Saunders NL (2012) Neuropsychological measures predict decline to Alzheimer's dementia from mild cognitive impairment. Neuropsychology 26, 498-508.

[5] Aretouli E, Okonkwo OC, Samek J, Brandt J (2011) The fate of the 0.5s: Predictors of 2-year outcome in mild cognitive impairment. J Int Neuropsychol Soc 17, 277-288.

[6] Nordlund A, Rolstad S, Klang O, Edman A, Hansen S, Wallin A (2010) Two-year outcome of MCI subtypes and aetiologies in the Goteborg MCI study. J Neurol Neurosurg Psychiatry 81, 541-546.

[7] Henry MS, Passmore AP, Todd S, McGuinness B, Craig D, Johnston JA (2013) The development of effective biomarkers for Alzheimer's disease: A review. Int J Geriatr Psychiatry 28, 331-340.

[8] Markesbery WR, Schmitt FA, Kryscio RJ, Davis DG, Smith CD, Wekstein DR (2006) Neuropathologic substrate of mild cognitive impairment. Arch Neurol 63, 38-46.

[9] Morris JC, Price AL (2001) Pathologic correlates of nondemented aging, mild cognitive impairment, and early-stage Alzheimer's disease. J Mol Neurosci 17, 101-118.

[10] Haroutunian V, Perl DP, Purohit DP, Marin D, Khan K, Lantz M, Davis K L, Mohs RC (1998) Regional distribution of neuritic plaques in the nondemented elderly and subjects with very mild Alzheimer disease. Arch Neurol 55, 1185-1191

[11] Mattsson N, Zetterberg H, Hansson O, Andreasen N, Parnetti L, Jonsson M, Herukka SK, van der Flier WM, Blankenstein MA, Ewers M, Rich K, Kaiser E, Verbeek M, Tsolaki M, Mulugeta E, Rosen E, Aarsland D, Visser PJ, Schroder J, Marcusson J, de Leon M, Hampel H, Scheltens P, Pirttila T, Wallin A, Jonhagen ME, Minthon L, Winblad B, Blennow $\mathrm{K}$ (2009) CSF biomarkers and incipient Alzheimer disease in patients with mild cognitive impairment. JAMA 302, 385-393.

[12] Colciaghi F, Marcello E, Borroni B, Zimmermann M, Caltagirone C, Cattabeni F, Padovani A, Di Luca, M (2004) Platelet APP, ADAM 10 and BACE alterations in the early stages of Alzheimer disease. Neurology 62, 498-501

[13] Tang K, Hynan LS, Baskin F, Rosenberg RN (2006) Platelet amyloid precursor protein processing: A bio-marker for Alzheimer's disease. J Neurol Sci 240, 53-58.

[14] Johnston JA, Liu WW, Coulson DT, Todd S, Murphy S, Brennan S, Foy CJ, Craig D, Irvine GB, Passmore AP (2008) Platelet beta-secretase activity is increased in Alzheimer's disease. Neurobiol Aging 29, 661-668.

[15] Liu WW, Todd S, Craig D, Passmore AP, Coulson DT, Murphy S, Irvine GB, Johnston JA (2007) Elevated platelet beta-secretase activity in mild cognitive impairment. Dement Geriatr Cogn Disord 24, 464-468.

[16] Gorham P, Bark N, Bjorkhem I, Meaney S, Crisby M (2010) Platelet alpha- and beta- secretase activities are not significantly affected by dementia or mild cognitive impairment in Swedish patients. Curr Alzheimer Res 7, 134-139

[17] Grimm MO, Grimm HS, Tomic I, Beyreuther K, Hartmann T, Bergmann C (2008) Independent inhibition of Alzheimer disease beta- and gamma-secretase cleavage by lowered cholesterol levels. J Biol Chem 283, 11302-11311.

[18] Liu WW, Todd S, Coulson DT, Irvine GB, Passmore AP, McGuinness B, McConville M, Craig D, Johnston JA (2009) A novel reciprocal and biphasic relationship between membrane cholesterol and beta-secretase activity in SH-SY5Y cells and in human platelets. J Neurochem 108, 341-349.

[19] Folstein MF, Folstein SE, McHugh PR (1975) 'Mini-mental state'. A practical method for grading the cognitive state of patients for the clinician. J Psychiatr Res 12, 189-198.

[20] Mioshi E, Dawson K, Mitchell J, Arnold R, Hodges JR (2006) The Addenbrooke's cognitive examination revised (ACE-R): A brief cognitive test battery for dementia screening. Int $J$ Geriatr Psychiatry 21, 1078-1085.

[21] Nelson HE, O'Connell A (1978) Dementia: The estimation of premorbid intelligence levels using the new adult reading test. Cortex 14, 234-244

[22] Kluger A, Ferris SH, Golomb J, Mittelman MS, Reisberg B (1999) Neuropsychological prediction of decline to dementia in nondemented elderly. J Geriatr Psychiatry Neurol 12, 168179

[23] Royall DR, Cordes JA, Polk M (1998) CLOX: An executive clock drawing task. J Neurol Neurosurg Psychiatry 64, 588594

[24] Stroop J (1935) Studies of interference in serial verbal reactions. J Exp Psychol 18, 643-661

[25] Burgess PW, Shallice T (1997) The Hayling and Brixton tests, Thames Valley Test Company, Thurston, Suffolk.

[26] D'Elia LF, Satz PJ, Uchiyama CL, White T (1996) Color trails test. Professional manual, psychological assessment resources, Odessa, FL.

[27] Benton AL, Hamsher SK, Sivan AB (1983) Multilingual aphasia examination (2nd edition). AJA Associates, Iowa City, IA.

[28] Gelinas I, Gauthier L, McIntyre M, Gauthier S (1999) Development of a functional measure for persons with Alzheimer's disease: The disability assessment for dementia. Am J Occup Ther 53, 471-481.

[29] Petersen RC, Smith GE, Waring SC, Ivnik RJ, Tangalos EG, Kokmen E (1999) Mild cognitive impairment: Clinical characterization and outcome. Arch Neurol 56, 303-308

[30] Nordlund A, Rolstad S, Hellstrom P, Sjogren M, Hansen S, Wallin A (2005) The Goteborg MCI study: Mild cognitive impairment is a heterogeneous condition. J Neurol Neurosurg Psychiatry 76, 1485-1490.

[31] McKhann G, Drachman D, Folstein M, Katzman R, Price D, Stadlan E (1984) Clinical diagnosis of Alzheimer's disease: Report of the NINCDS-ADRDA work group under the auspices of department of health and human services task force on Alzheimer's disease. Neurology 34, 939-944

[32] Bermejo-Bescos P, Martin-Aragon S, Jimenez-Aliaga K, Benedi J, Felici E, Gil P, Ribera JM, Villar AM (2013) Processing of the platelet amyloid precursor protein in the mild cognitive impairment (MCI). Neurochem Res 38, 1415-1423.

[33] Decourt B, Sabbagh MN (2011) BACE1 as a potential biomarker for Alzheimer's disease. JAlzheimers Dis 24(Suppl 2), 53-59. 